\title{
Characterization of the liver $X$ receptor-dependent regulatory mechanism of goat stearoyl-coenzyme $A$ desaturase 1 gene by linoleic acid
}

\author{
D. W. Yao, ${ }^{*}$ J. Luo, ${ }^{* 1}$ Q. Y. He, ${ }^{*}$ J. Li, † H. Wang, ${ }^{*}$ H. B. Shi,‡ H. F. Xu, ${ }^{\star}$ M. Wang, ${ }^{*}$ and J. J. Loor ${ }^{1}$ \\ *Shaanxi Key Laboratory of Molecular Biology for Agriculture, College of Animal Science and Technology, Northwest A\&F University, Yangling, \\ Shaanxi, P. R. China 712100 \\ †College of Animal Science and Technology, Henan University of Animal Husbandry and Economy, Zhengzhou, Henan, P. R. China 450046 \\ $\ddagger$ College of Animal Sciences, Zhejiang Sci-Tech University, Hangzhou, P. R. China 310058 \\ $\S$ Mammalian NutriPhysioGenomics, Department of Animal Sciences and Division of Nutritional Sciences, University of Illinois, Urbana 61801
}

\section{ABSTRACT}

Stearoyl-coenzyme A desaturase 1 (SCD1) is a key enzyme in the biosynthesis of palmitoleic and oleic acid. Although the transcriptional regulatory mechanism of $S C D 1$ via polyunsaturated fatty acids (PUFA) has been extensively explored in nonruminants, the existence of such mechanism in ruminant mammary gland remains unknown. In this study, we used goat genomic DNA to clone and sequence a 1,713-bp fragment of the $S C D 15^{\prime}$ flanking region. Deletion assays revealed a core region of the promoter located between -415 and $-109 \mathrm{bp}$ upstream of the transcription start site, and contained the highly conserved PUFA response region. An intact PUFA response region was required for the basal transcriptional activity of $S C D 1$. Linoleic acid reduced endogenous expression of $S C D 1$ and sterol regulatory element binding factor-1 (SREBF1) in goat mammary epithelial cells. Further analysis indicated that both the sterol response element (SRE) and the nuclear factor $\mathrm{Y}$ (NF-Y) binding site in the SCD1 promoter were responsible for the inhibition effect by linoleic acid, whereas the effect was abrogated once NF-Y was deleted. In addition, SRE and NF-Y were partly responsible for the transcriptional activation induced via the liver $\mathrm{X}$ receptor agonist T0901317 (Sigma-Aldrich, St. Louis, $\mathrm{MO})$. When goat mammary epithelial cells were cultured with linoleic acid, addition of T0901317 markedly increased $S C D 1$ transcription in controls, but had no effect on cells with a deleted SRE promoter. These results demonstrated that linoleic acid can regulate $S C D 1$ expression at the transcriptional level through SRE and $\mathrm{NF}-\mathrm{Y}$ in a liver $\mathrm{X}$ receptor-dependent fashion in the goat mammary gland.

Key words: stearoyl-CoA desaturase 1 promoter, $S R E B F 1$, linoleic acid, transcriptional regulation

\footnotetext{
Received November 5, 2015.

Accepted January 13, 2016.

${ }^{1}$ Corresponding authors: luojun@nwsuaf.edu.cn and jloor@illinois.
} edu

\section{INTRODUCTION}

Goat milk is rich in MUFA and PUFA, therefore conferring a special nutritional value (Haenlein, 2004). The high content of MUFA and PUFA in goat milk are beneficial for human health, including the treatment of cardiovascular conditions and hypercholesterolemia or hyperlipemia (Noakes et al., 1996; Addrizzo, 2004; Haenlein, 2004; Bhattacharya et al., 2006). Conjugated linoleic acids, which are a metabolic by-product of rumen hydrogenation and rich in goat milk, have been proven to be a potent inhibitor for tumor growth (Williams, 2000; Haenlein, 2004; Beppu et al., 2006). In lactating ruminants, the mammary enzyme stearoylcoenzyme A desaturase 1 (encoded by $S C D 1$ ) plays a pivotal role in the synthesis of milk MUFA and specific CLA isomers by introducing a cis double bond between carbons 9 and 10 of 18:0 or trans-11 18:1. Therefore, exploring the regulatory mechanisms of $S C D 1$ in goat mammary epithelial cells (GMEC) might, in the long term, help develop strategies for manipulating the concentration of beneficial fatty acids in ruminant milk.

In rodents it is well-established that dietary PUFA suppress the transcription of several genes encoding key enzymes involved in biochemical pathways of fat synthesis, including fatty acid synthase, acetyl-CoA carboxylase $\alpha$, and stearoyl-coenzyme A desaturase. For instance, diets rich in linoleic acid decreased the expression of hepatic SCD1 in neonatal Balb/c mice (DeWille and Farmer, 1993). Exogenous linoleic acid also decreased the expression of $S C D 1 \mathrm{mRNA}$ in rat hepatocytes (Landschulz et al., 1994) and the activity of the human SCD1 promoter, which contains 609 bp upstream of the transcription start site (Zhang et al., 2001).

The findings in nonruminants raise the possibility that the PUFA effect on mammary $S C D 1$ expression reported previously in nutritional studies with ruminants could occur through similar mechanisms. Research with dairy cows indicated a tight and complex regulation of $S C D 1$ gene expression in response to PUFA. For 
Table 1. Primers used for isolation and deletion of goat $S C D 1$ promoter constructs

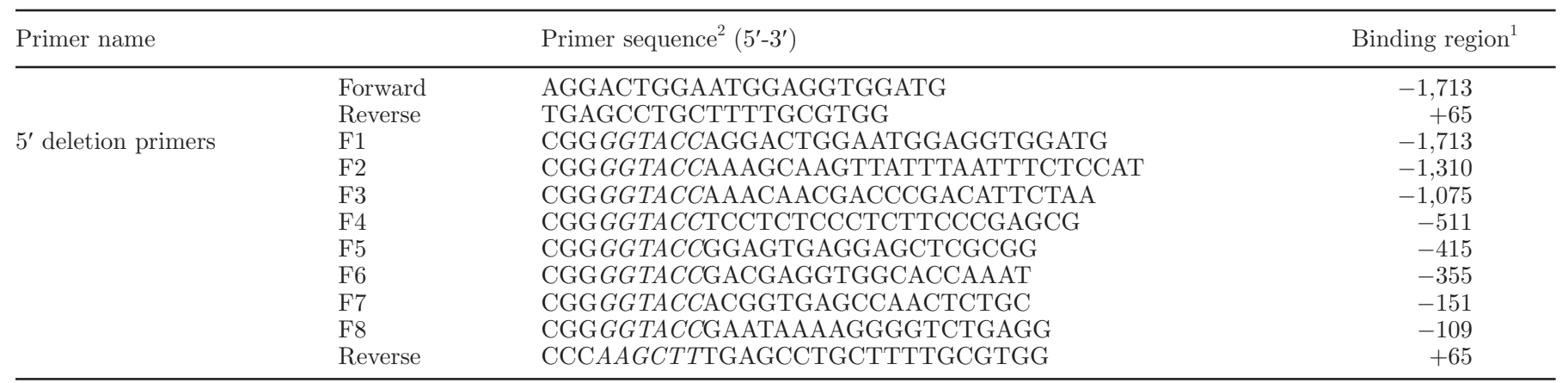

${ }^{1}+$ and - indicate the number of bases upstream $(-)$ and downstream $(+)$ from the transcriptional start site.

${ }^{2}$ Restriction enzyme sites are shown in italics.

instance, abomasal infusion or intravenous injection of trans-10,cis-12 CLA decreased mammary SCD1 gene transcription (Baumgard et al., 2002; Gervais et al., 2009). In contrast, feeding saturated long-chain fatty acids or a milk fat-depressing diet induced a sustained upregulation of $S C D 1$ transcription in bovine mammary tissue (Invernizzi et al., 2010). In goats, a duodenal infusion of trans-10,cis-12 CLA led to the reduction in the delta-9 desaturase index of milk fat (de Andrade and Schmidely, 2006).

Waters et al. (1997) localized genetic regulatory elements for PUFA in the mouse SCD1 promoter, which contains consensus binding sites for the transcription factors specificity protein 1 (Sp1), sterol regulatory element-binding proteins (SREBP), and nuclear factorY (NF-Y; Waters et al., 1997). Subsequent work demonstrated that PUFA modulates SCD1 gene expression through several mechanisms, leading to a decrease in the nuclear abundance of SREBP-1c (Ou et al., 2001; Botolin et al., 2006). Sterol regulatory element-binding protein, which in nonruminants responds to insulin and activates the $S C D 1$ promoter, was confirmed to play a central role in the transcriptional activation of $S C D 1$ (Bené et al., 2001; Espenshade, 2006). Previous data also indicated that PUFA might repress the lipogenic gene expression by reducing NF-Y binding on the FASN promoter (Teran-Garcia et al., 2002). Besides SREBP$1 \mathrm{c}$, at least in nonruminants, the transcription factor liver-X-receptor ( $\mathbf{L X R}$ ) is also involved in the control of lipogenesis in an SREBP-dependent and -independent manner (Ulven et al., 2005).

Although the transcriptional regulation of $S C D 1$ by PUFA in vivo and in vitro is well-known in nonruminants, such knowledge is nonexistent in a ruminant species such as the goat, whose mammary gland during lactation becomes the central lipogenic tissue. The main goal of our study was to clone and characterize the goat $S C D 1$ promoter, and evaluate the transcriptional regulatory mechanisms elicited by PUFA in GMEC.

\section{MATERIALS AND METHODS}

\section{Cloning of the SCD1 Promoter Region and Bioinformatics Analysis}

The 5' flanking sequence of the $S C D 1$ gene was amplified from goat genomic DNA that was extracted from blood samples of 5 goats using a genomic DNA extraction kit (Tiangen, Beijing, China). Primers listed in Table 1 were designed using information from the Yunnan black goat genome and Bos taurus genome. We used PrimeSTAR HS DNA Polymerase (Takara Bio Inc., Otsu, Japan) to amplify the sequence according to the manufacturer's protocols. Amplified products were cloned into the pMD19-T vector (Takara Bio Inc.) and sequenced by Invitrogen (Shanghai, China).

The promoter region was predicted using Promoter 2.0 (http://www.cbs.dtu.dk/services/Promoter/) and compared with that of bovine, human, and rat using the BioXM 2.6 software (Nanjing Agricultural University, Nanjing, China). The putative transcription factor binding sites were initially predicted using the following online tools: MatInspector (http://www.genomatix. de/), AliBata 2.1 (http://www.gene-regulation.com/ pub/programs.html), the JASPAR database (http:// jaspar.genereg.net/), and the Peroxisome Proliferator Response Elements Search (http://www.classicrus. $\mathrm{com} / \mathrm{PPRE} /$ ).

\section{Deletion Analysis and Plasmid Construction}

The resulting fragment of the $S C D 1$ promoter region was gel-purified using a Tiangen quick gel extraction kit (Tiangen), and employed in a second PCR to introduce $K p n I$ and HindIII (Takara Bio Inc.) restriction sites to insert into the upstream of the luciferase gene using the pGL-3 Basic vector (Promega, Madison, WI). Similarly, the $5^{\prime}$ deletion plasmids were constructed using the fragments derived from primers that hybridize at 
positions $-1,713,-1,310,-1,075,-511,-415,-355$, -151 , and -109 . The common downstream primer was designed at position +65 . All of the fragments inserted into the plasmids were confirmed by DNA sequencing.

\section{Site-Directed Deletion}

To introduce site-directed deletions of the binding sites for Sp1, SRE, and NF-Y in the SCD1 promoter region, an overlap PCR strategy was used. First, to generate partially overlapping fragments, the primers listed in Table 2 were used using the wild-type insert as template. Then, the 2 DNA fragments with the designated mutations were gel-purified and used in a second PCR as templates to generate a third DNA fragment. The Sp1, SRE, and NF-Y mutants were constructed using pGL- $(-511 /+65)$ constructs as templates. The final overlapping fragments were then digested with $K p n \mathrm{I}$ and HindIII to generate mutant constructs. The presence of the correct insert was verified by DNA sequencing.

\section{Cell Culture and Transfection}

Goat primary GMEC were harvested from peaklactation Xinong Saanen dairy goats, as described previously, and the cell culture procedures were according to our previous publications (Lin et al., 2013; Zhu et al., 2015). The GMEC were cultured in a basal F-12 (DMEM/F12; Hyclone, Logan, UT) medium composed of $5 \mu \mathrm{g} / \mathrm{mL}$ of insulin (Sigma-Aldrich, St. Louis, MO), $100 \mathrm{U} / \mathrm{mL}$ of penicillin/streptomycin (Harbin Pharmaceutical Group Co. Ltd., Harbin, China), $10 \mathrm{ng} / \mathrm{mL}$ of epidermal growth factor 1 (EGF-1, Invitrogen), $1 \mu \mathrm{g} /$ $\mathrm{mL}$ of hydrocortisone (Sigma-Aldrich), and 10\% fetal bovine serum (Hyclone) in an environment of $5 \% \mathrm{CO}_{2}$ in air at $37^{\circ} \mathrm{C}$; culture medium was changed every 24 h. The GMEC isolated from the 3 goats were cultured individually to passage 5 and then pooled before starting the experiment. In the cell culture medium, the concentration of glucose and acetate was $3.15 \mathrm{~g} / \mathrm{L}$ and $0.04 \mathrm{~m} M$, respectively. To induce lactogenesis, GMEC were cultured with prolactin $(2 \mu \mathrm{g} / \mathrm{mL}$; Sigma-Aldrich) via supplementing the basal medium for $48 \mathrm{~h}$ before the experiments were performed, as described previously (Kadegowda et al., 2009).

The cells were seeded in 48-well plates (Corning Inc., Corning, NY) and cultured overnight or until 80 to $90 \%$ confluence. Then, the GMEC were transientlytransfected with $300 \mathrm{ng}$ of total DNA per well using X-tremeGENE HP DNA Transfection Reagent (Roche Ltd., Shanghai, China) following the manufacturer's instructions. Renilla luciferase vector (pRL-TK, Promega) was used as an internal control to normalize transfection efficiency, and the ratio of pGL-SCD1 compare with pRL-TK was 30:1. Linoleic acid was prepared according to a previous study (Kadegowda et al., 2009). After transfection for $12 \mathrm{~h}$, cell culture medium was changed with starvation medium free of fetal bovine serum and incubated for $12 \mathrm{~h}$. Then, linoleic acid $(100 \mu \mathrm{mol} / \mathrm{L})$ and BSA $(1 \mathrm{~g} / \mathrm{L})$ were added to the fetal bovine serum-free culture medium before incubation of cells for $16 \mathrm{~h}$. As for the treatment with T0901317 (T09), a specific agonist of LXR (dissolved in dimethyl sulfoxide; Sigma-Aldrich), the concentration of T09 was $1 \mu M$ and used for incubations lasting for $24 \mathrm{~h}$ with dimethyl sulfoxide incubated separately to serve as a control according to our previous experiments (Wang et al., 2012). Data presented are based on 3 individual experiments.

Table 2. Primers used for site-directed deletion of goat SCD1 promoter constructs

\begin{tabular}{ll}
\hline Primer name $^{1}$ & Primer sequence $^{2}\left(5^{\prime}-3^{\prime}\right)$ \\
\hline F4 & CGGGGTACCTCCTCTCCCTCTTCCCGAGCG \\
Sp1 & GCGGC \\
Sp1-dele & GGAGGGGGAGTGAGGAGCTCAGAGGGAACAG \\
Sp1-anti-dele & CGCAATCTGCTGTTCCCTCTGAGCTCCTCA \\
SRE & AGCAGATTGCG \\
SRE-dele & GCCGTCGCCATTGGCTCGGGTTCCCTCTGC \\
SRE-anti-dele & AGCTCGCGGCAGAGGGAACCCGAGCCAATG \\
NF-Y & CAATGGCG \\
NF-Y-dele & AACAGCAGATTGCGCCGAGCACGGCAGGACG \\
NF-Y-anti-dele & GTGCCACCTCGTCCTGCCGTGCTCGGCGCAA \\
SRE/NF-Y-dele & CGCGGCAGAGGGAACCCGAGCACGGCAGGACG \\
SRE/NF-Y-anti-dele & GGTGCCACCTCGTCCTGCCGTGCTCGGGTCCC \\
Sp1/SRE/NF-Y-dele & TGCCGTGCTCGGGTTCCCTCTGAGCTCCTCACTCC \\
Sp1/SRE/NF-Y-anti-dele & GGAGGGGGAGTGAGGAGCTCAGAGGGAACCCGAG \\
Reverse & CCCAAGCTTTGAGCCTGCTTTTGCGTGG \\
${ }^{1}$ Sp1 $=$ specificity protein $1 ;$ SRE $=$ & sterol response element; NF-Y = nuclear factor Y. \\
${ }^{2}$ Deletion letters are shown in bold font. Restriction enzyme sites are shown in italic font. &
\end{tabular}




\section{Luciferase Assays}

After $48 \mathrm{~h}$ of transfection, cells were washed twice with PBS and lysed with Promega passive lysis buffer to measure luciferase activity. Reporter activity was measured using the Dual-Luciferase Reporter assay kit (Promega) on a Fluoroskan Ascent apparatus (Thermo Fisher Scientific, Waltham, MA). The relative luciferase activity was calculated as the ratio of Firefly (Promega) compared with Renilla luciferase activity.

\section{RNA Extraction and Quantitative Real-Time PCR}

We used the RNAprep Pure Cell/Bacteria Kit (Tiangen) to extract total RNA from GMEC. The cDNA was synthesized using the PrimeScript RT kit (Perfect Real time, Takara Bio Inc.) following the manufacturer's protocols. The real-time quantitative PCR was performed using SYBR Green (SYBR Premix Ex TaqII, Perfect Real Time, Takara Bio Inc.) on a CFX96 Real-Time PCR Detection System (Bio-Rad, Hercules, CA) under the following conditions: $95^{\circ} \mathrm{C}$ for $4 \mathrm{~min}$, followed by 40 cycles at $95^{\circ} \mathrm{C}$ for $15 \mathrm{~s}, 60^{\circ} \mathrm{C}$ for $30 \mathrm{~s}$, and $72^{\circ} \mathrm{C}$ for 30 s. The presence of a single PCR product was confirmed by the association protocol using incremental temperatures to $95^{\circ} \mathrm{C}$ for $15 \mathrm{~s}$ and $65^{\circ} \mathrm{C}$ for $5 \mathrm{~s}$.

The internal controls were ubiquitously expressed transcript $(U X T)$, mitochondrial ribosomal protein L39 (MRPL39), and ribosomal protein S9 (RPS9) to normalize of targeted mRNA profiles (Ramunno et al., 2005; Bionaz and Loor, 2007). The primers of SCD1, SREBF1, UXT, MRPL39, and RPS9 are listed in Table 3. Every sample was analyzed in sextuplicate.

\section{Statistical Analysis}

Results were analyzed using SPSS 19.0 (SPSS Inc., IBM Corp., Armonk, NY) and expressed as mean \pm standard error of means of at least 3 independent experiments. Data of real-time quantitative PCR were analyzed using $2^{-\Delta \Delta \mathrm{Ct}}$ method relative to the control, where $\mathrm{Ct}$ is the cycle threshold. Results were considered statistically significant at $P<0.05$ using a Student's $t$-test (unpaired and 2-tailed) for a one-way ANOVA.

\section{RESULTS}

\section{Cloning, Sequencing, and Characterization of the Goat SCD1 Promoter}

We generated a 1,778-bp 5' flanking sequence (GenBank accession no. KT266815) by PCR containing 1,713 bp upstream of the transcription start site (TSS; +1 ) and part of the first exon (Figure 1A). A TATA box-like sequence was located at $49 \mathrm{bp}$ upstream of the TSS. Bioinformatics analysis revealed a series of consensus binding sites for transcription factors such as NF-Y (-361 bp), Sp1 (-164, -249, -396, and -528 bp), activating protein $2(-150,-324$, and $-496 \mathrm{bp})$, peroxisome proliferator-activated receptor gamma $(-173$ and $-418 \mathrm{bp})$, SRE (-376 bp), CCAAT-enhancer-binding site $(-289 \mathrm{bp})$, and estrogen receptor $(-204$ and -468 bp; Figure 1B). Furthermore, an alignment of this sequence with the human (AF320307) and mouse SCD1 promoter sequence (M21280) revealed that the PUFA response regions (PUFA-RR) are highly conserved among human, mouse, and goat (Figure 1C).

\section{Identification of the Promoter Region and Essential Elements}

The region responsible for basal transcription activity of the $S C D 1$ gene promoter was confirmed by producing progressive deletions from 1,778 bp upstream of $5^{\prime}$, and cloning into a luciferase reporter system. These fragments extended from a common 3 ' start point $(+65)$ in the promoter and extended to the $5^{\prime}$ upstream of the transcriptional start site, including $-1,713,-1,310$, $-1,075,-511,-415,-355,-151$, and $-109 \mathrm{bp}$, as depicted in Figure 2A. These truncated constructs were

Table 3. Characteristics of primer pairs used and efficiency of reaction in the real-time quantitative PCR

\begin{tabular}{|c|c|c|c|c|}
\hline Gene & Accession no. & Primer sequence $\left(5^{\prime}-3^{\prime}\right)$ & Efficiency & Source \\
\hline$S C D 1$ & GU947654 & $\begin{array}{l}\text { F: CCATCGCCTGTGGAGTCAC } \\
\text { R: GTCGGATAAATCTAGCGTAGCA }\end{array}$ & 2.10 & Shi et al., 2013 \\
\hline$S R E B F 1$ & HM443643.1 & $\begin{array}{l}\text { F: ACGCCATCGAGAAACGCTAC } \\
\text { R: GTGCGCAGACTCAGGTTCTC }\end{array}$ & 2.04 & Shi et al., 2013 \\
\hline
\end{tabular}


then transiently transfected into GMEC and the relative luciferase activity was detected to explore the core area involved in regulating transcription of the gene. The DAPI (4',6-diamidino-2-phenylindole) staining results showed that the viability of GMEC was almost not influenced at $24 \mathrm{~h}$ by the transfection protocol (Supplemental Figure S1; http://dx.doi.org/10.3168/ jds.2015-10601). The luciferase activity of the SCD1

A

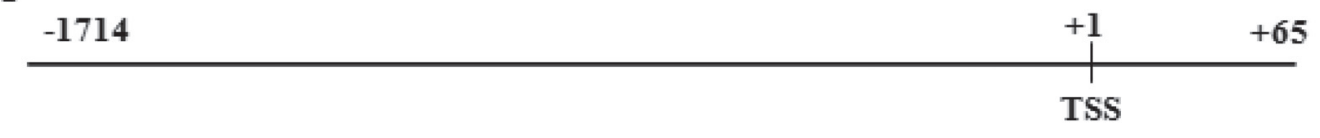

B

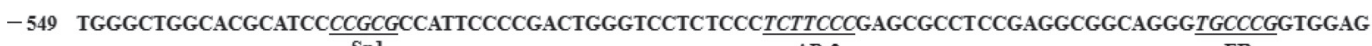

Spl AP-2 ER

- 461 GCCCAGCGGCCGGTGTAAGAGAAGCCGAGGAGAAAGGGAGGGGAGGGGGAGTGAGGAGCTCGCGGCAGAGGGAACAGCAGATTGCG

PPRE

-375 CCGAG $\underline{C C A A T G G C G A C G G C A G G A C G A G G T G G C A C C A A A T T C C C T T C G G C C A A T G A C G C G C C A G A G T C T A C A G A A G C C C A T} \underline{T A G C A T T}$

NF-Y

AP-2

C/EBP

-288 TCCCCAGGGGCAGGGGCAGAGGCAGGGGCTGCGGCGGCCAAACCGCGGTGTGTCTGCAGCATCCAGTTCTCGCTTCTCCTGCCCCCAG

Spl

ER

-200 CACGCCTCGGCGCTCTGTCTCCTCCCCTCTCCCGCCCATGCGGATCTCCCACGGTGAGCCAACTCTGCGCACTTTGCCCCTTGTTGGCAG PPRE Spl AP-2

- 110 CGAATAAAAGGGGTCTGAGGAAATACGAGACACAGTCACCGGCTGCCAGCGCTAGCCTTTAAATCCCCAGCACAGCAGGTCGGGTCCG

-22 GACACCGATCCAGCGCGCAGCGTGCAGCGGAAGGTCCCGAGCACAGCGCCGCGGATCGCCACGCATCGCCACGCAAAAGCAGGCTCA TSS

C

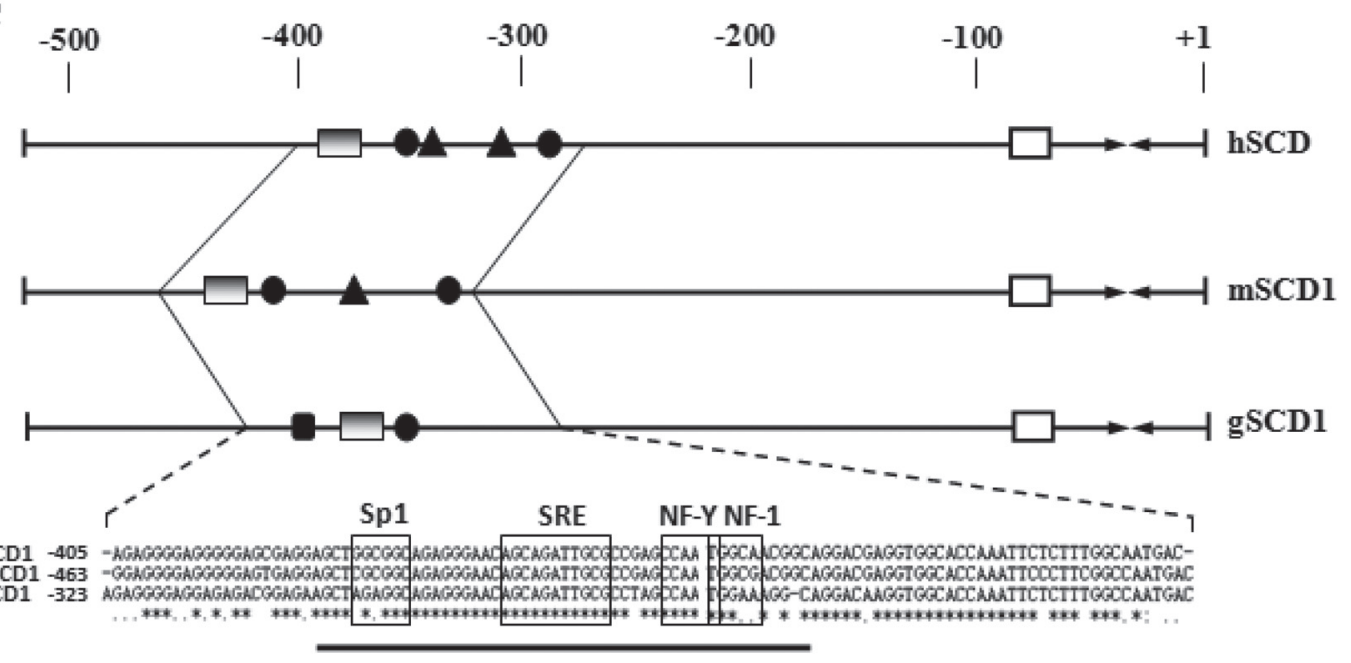

PUFA Response Region

SP1

Figure 1. Bioinformatics analysis and characterization of the stearoyl-coenzyme A desaturase 1 (SCD1) promoter region. (A) Schematics of the $S C D 1$ gene $5^{\prime}$ flanking region; +1 represents the transcription start site (TSS). (B) Predicted putative transcription factors in the $5^{\prime}$ flanking region of goat $S C D 1$. The consensus sequence transcription factor (TF) binding sites are underlined with the corresponding names of the TF. (C) A schematic representation of alignment analysis of the 5' flanking region of the human and mouse SCD1 (cloning) compared with goat. The top scale indicates the nucleotide upstream of the TSS. Putative polyunsaturated fatty acid response region (PUFA-RR) including transcription factors specificity protein 1 (Sp1), sterol response element (SRE), and nuclear factor Y (NF-Y) are denoted. An asterisk (*) denotes exact base matches among species. $\mathrm{C} / \mathrm{EBP}=\mathrm{CCAAT}$-enhancer-binding site. 
gene promoter was highest when the fragment deleted reached up to position -511 bp (pGL $-511 /+65)$, and slightly declined at position $-415 \mathrm{bp}$ (pGL $-415 /+65)$. A significant decrease in promoter activity was detected using the construct pGL- $(-355 /+65)$. Further deletions almost abolished the promoter activity in the construct pGL- $(-109 /+65)$. Altogether, these data demonstrated that the region between -415 and $-355 \mathrm{bp}$ in the pGL$(-415 /+65)$ contained essential cis-functional elements required for maximal $S C D 1$ transcriptional activation, and the core region of the $S C D 1$ promoter spans from -415 to -109 bp to maintain basal transcriptional activity of the gene.

\section{PUFA-RR Influences SCD1 Promoter Activity}

The PUFA-RR is highly conserved among species (Figure 1C). The consensus binding sites for Sp1, SRE, and NF-Y within the PUFA-RR may play an important role in maintaining the transcriptional activity of the $S C D 1$ gene promoter. To evaluate their roles, we constructed site-deleted luciferase reporter vectors for Sp1, SRE, and NF-Y, and transiently transfected them into the GMEC for promoter activity. As shown in Figure 2B, the site-deleted Sp1 did not elicit a significant effect on $S C D 1$ gene promoter activity $(P>0.05)$. However, the promoter activity was markedly decreased $(P<$

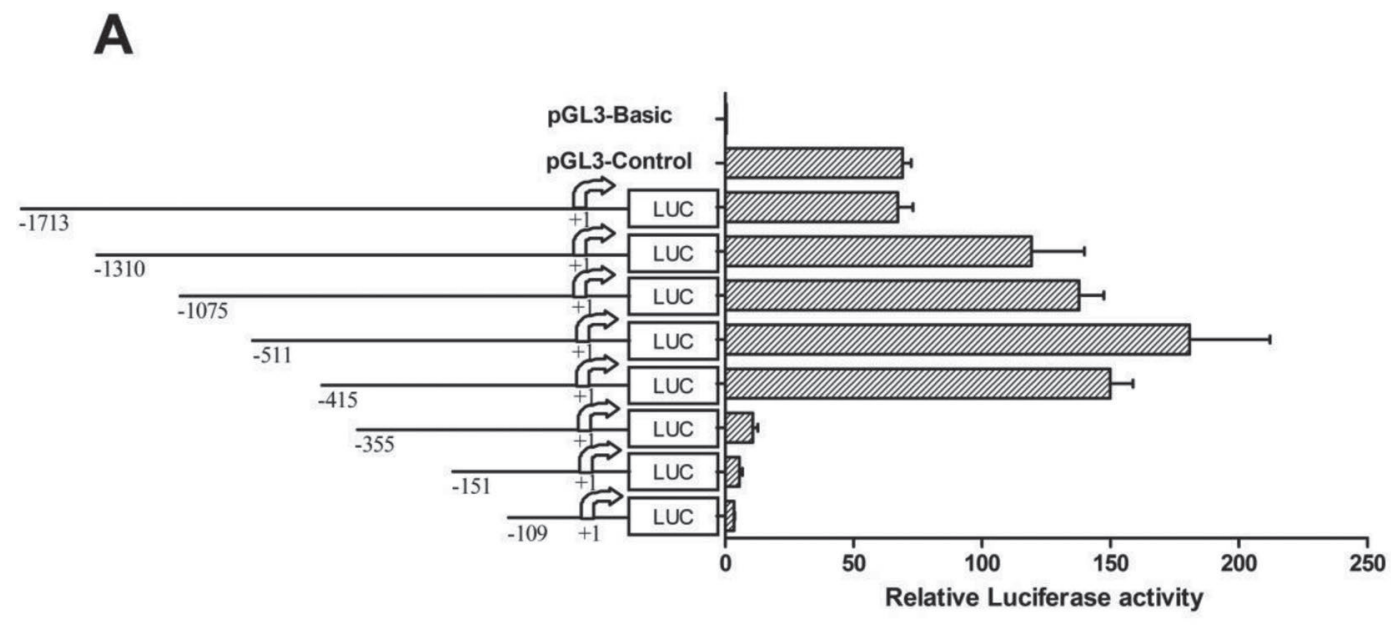

B

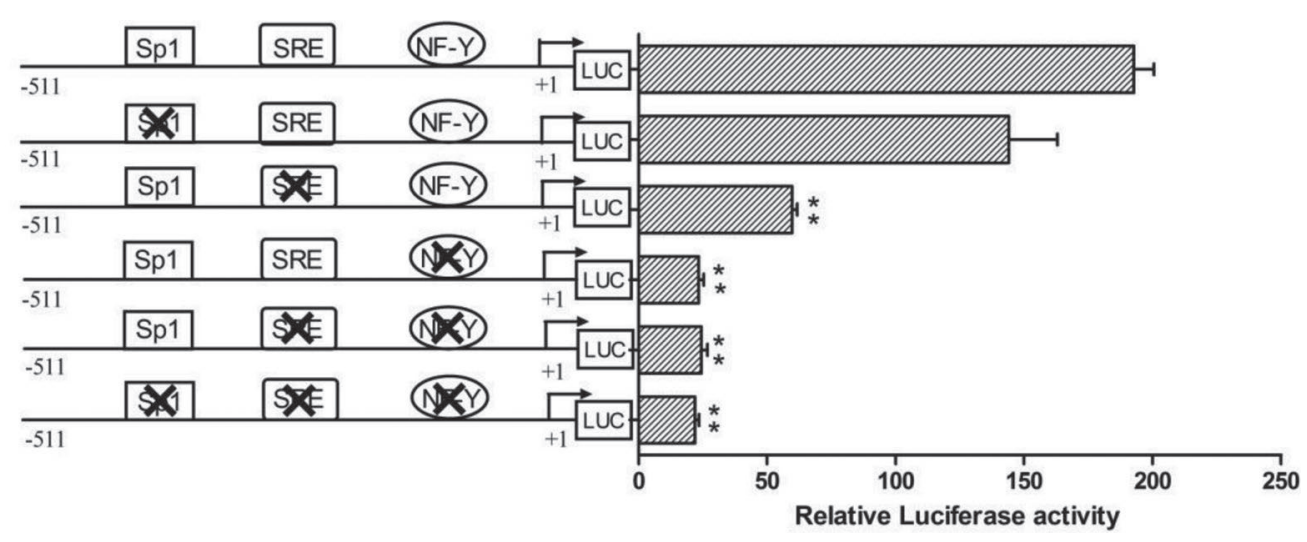

Figure 2. Determination of the core region and required transcription factor (TF) binding sites responsible for basal transcriptional activity of the goat stearoyl-coenzyme A desaturase 1(SCD1) promoter. (A) Progressive deletion fragments of goat SCD1 promoter were generated as described in Materials and Methods. Equimolar amounts of constructs with pGL-Basic and pGL-control (Promega, Madison, WI) were transiently transfected into goat mammary epithelial cells (GMEC). (B) Relative luciferase activity after deletion of transcription binding sites for transcription factors specificity protein 1 (Sp1), sterol response element (SRE), and nuclear factor Y (NF-Y) on the $S C D 1$ promoter. Data for luciferase activity were normalized relative to luciferase activity (firefly/Renilla) and presented as mean \pm SEM. All experiments were performed in triplicate and repeated 3 times $(\mathrm{n}=9) .{ }^{*} P<0.05 ;{ }^{*} P<0.01$. 
0.01) when the SRE was deleted. Furthermore, deletion of NF-Y or the combination of SRE and NF-Y in the $S C D 1$ gene promoter region nearly diminished its activity to $12 \%(P<0.01)$ of that of the control wild-type construct pGL- $(-511 /+65)$. Collectively, these results suggested that SRE and NF-Y play a critical role in regulating transcription of $S C D 1$.

\section{Linoleic Acid Repressed SCD1 Expression and Promoter Activity}

In addition, the mRNA expression of $S C D 1$ was significantly reduced by linoleic acid compared with eicosapentaenoic acid or arachidonic acid (Supplemental Figure S2; http://dx.doi.org/10.3168/jds.2015-10601). The DAPI staining results showed that the viability of GMEC was influenced very little by the linoleic acid treatment (Supplemental Figure S3; http://dx.doi. org/10.3168/jds.2015-10601). Therefore, it was particularly important to evaluate the effect that linoleic acid would have on the goat $S C D 1$ gene expression and promoter activity. As depicted in Figure $3 \mathrm{~A}$ and $3 \mathrm{~B}$, the relative mRNA expression of $S R E B F 1$ and $S C D 1$ decreased dramatically $(P<0.05)$ after GMEC were treated with $100 \mu M$ linoleic acid for $24 \mathrm{~h}$. Consistent with this effect, linoleic acid repressed the SCD1 gene promoter activity of constructs with a progressive deletion except for the pGL- $(-109 /+65)$. Collectively, the data indicated that the region responsive to linoleic acid is located within -511 to -109 bp of the SCD1 gene promoter.

To further identify the transcription factor binding sites involved in the regulation of $S C D 1$ by linoleic acid, the constructs with site-deleted consensus binding sites for Sp1, NF-Y, SRE, or their combination were transiently transfected into GMEC and incubated with linoleic acid for $16 \mathrm{~h}$. The relative luciferase results indicated that deletion of Sp1 or SRE could not eliminate the linoleic acid-mediated reduction of $S C D 1$ gene promoter activity $(P<0.01)$. In contrast, deletion constructs of NF-Y, NF-Y together with SRE, or a combination of NF-Y, SRE, and Sp1 completely abolished the repressive effect of linoleic acid (Figure 4). Taken together, these results indicated that SRE and $\mathrm{NF}-\mathrm{Y}$ were responsible for the influence of linoleic acid on the gene promoter $S C D 1$.

\section{Linoleic Acid Reduced SCD1 Gene Promoter Activity}

In rodents, $S C D 1$ is regulated by LXR through an LXR response element (LXRE) sequence in the $S C D 1$ gene promoter region through an SREBP-1-independent mechanism. As depicted in Figure 5A, although we did not observe a typical LXRE in the goat $S C D 1$ gene promoter, the LXR agonist T09 also could stimulate the transcriptional activity of $S C D 1(P<0.01)$. In addition, deletion of SRE could not abolish this effect. Similarly, the transcriptional activity of the SCD1 gene containing deletion of NF-Y and the combination of NF-Y and SRE also was upregulated by T09 $(P<$ 0.05). Therefore, the mechanism responsible for T09 stimulation may depend on LXRE or SRE in the SCD1 gene promoter.

To further evaluate the possible involvement of linoleic acid on $S C D 1$ transcriptional regulation mediated by $L X R$, we selected constructs without (wild type) or with an SRE-deleted SCD1 gene promoter. After transiently transfecting these constructs into GMEC, the cells were first treated with T09 for $48 \mathrm{~h}$ followed by incubation with linoleic acid for $16 \mathrm{~h}$. Although linoleic acid reduced the wild-type $S C D 1$ gene promoter activity, T09 could eliminate this effect and notably increased $(P<0.05)$ the transcriptional activity of $S C D 1$. In cells transfected with the SRE-deleted SCD1 gene promoter, the stimulatory effect of T09 on the $S C D 1$ gene promoter activity was abolished when linoleic acid was incubated. Collectively, these data suggested that LXR participates in the regulation of the $S C D 1$ gene promoter via linoleic acid mainly through the conserved SRE binding site.

\section{DISCUSSION}

The enzyme SCD is key for the formation of palmitoyl-CoA and stearoyl-CoA, which serve as important substrates for the synthesis of phospholipids, triacylglycerols, cholesterol, and wax esters (Goldman and Vagelos, 1961). However, regulation of its expression in the dairy goat mammary gland is poorly understood. In the present study, we isolated the $5^{\prime}$ flanking region of the goat $S C D 1$ gene and characterized the gene promoter for its basal transcription activity. Although alignment of sequences generated for the goat SCD1 promoter regions with the published human $S C D 1$ (accession number AF320307) and mouse SCD1 (accession number MSDS1) promoters demonstrated fairly low homology across species, the putative PUFA-RR located at -367 to -401 is highly conserved and agrees with a previous study (Zulkifli et al., 2010). In addition, our data showed that the fragment (from -415 to $+65 \mathrm{bp}$ ) containing the PUFA-RR exhibited a significant increase in the promoter activity. This region was previously reported to be important in maintaining the basal transcriptional activity of the bovine $S C D 1$ gene promoter (Keating et al., 2006). A slight increase in promoter activity was observed as the promoter region extended to $511 \mathrm{bp}$ upstream of the TTS. Therefore, 

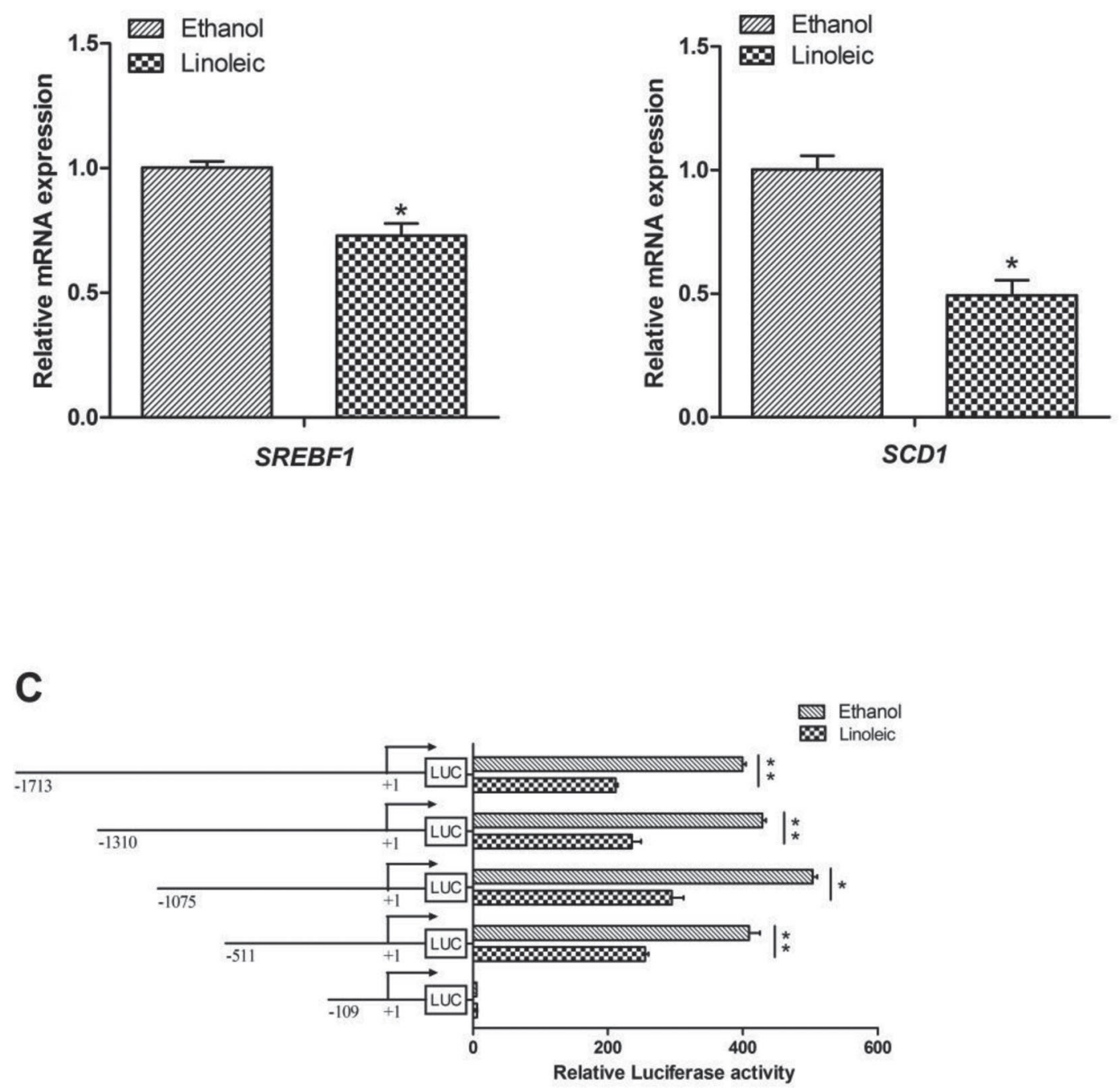

Figure 3. Effects of linoleic acid on the mRNA expression of stearoyl-coenzyme A desaturase 1 (SCD1) and sterol regulatory element binding factor-1 (SREBF1), and different lengths of $S C D 1$ promoter activity. Endogenous SREBF1 (A) and SCD1 (B) expression levels after treatment of GMEC with linoleic acid for $24 \mathrm{~h}$. (C) After different $5^{\prime}$ flanking deletion constructs of the $S C D 1$ promoter were transiently transfected into goat mammary epithelial cells (GMEC), linoleic acid was added in a serum-free medium with BSA and cultured for 24 h. Relative luciferase activity was detected as described in Materials and Methods. The experiments of mRNA expression were performed in sextuplicate and repeated 3 times $(\mathrm{n}=18)$, and the relative luciferase activity was performed in triplicate and repeated 3 times $(\mathrm{n}=9)$. Results are presented as mean \pm SEM for 3 individuals. ${ }^{*} P<0.05 ;{ }^{*} P<0.01$.

the core region of the goat $S C D 1$ gene promoter, which is required for the basal promoter activity, was located in the 415 bp upstream sequence.

The conserved putative PUFA-RR contains DNA recognition sites for Sp1, SRE, and NF-Y. In nonruminants, SREBP-1 has been proven to regulate the expression of $S C D 1$ through an SRE in its promoter region (Shimomura et al., 1998; Tabor et al., 1999). The role of SREBP-1 on the regulation of SCD1 in bovine was confirmed by use of small interfering (si)RNA for
SREBP-1 in Mac-T cells, which resulted in decreased SCD1 expression (Ma and Corl, 2012). However, it is known in nonruminants that transcriptional activation of SREBP requires additional transcription factors, including Sp1 and NF-Y. Activity of NF-Y is required for the transcriptional activation of mouse $S C D 1$ and $S C D 2$ promoters in response to cellular sterol deprivation (Tabor et al., 1999). Our study supports the idea that SRE and NF-Y are both essential in maintaining the basal transcriptional activity of the goat $S C D 1$ pro- 
moter. However, deletion of Sp1 revealed only a modest effect on basal promoter activity, which indicates that Sp1 plays a lesser role.

It has long been recognized in nonruminants that PUFA, both n-3 and n- 6 series, are potent inhibitors of SCD1 gene expression (Ntambi, 1999). In lactating cows, postruminal infusion studies first proved that a mixture of CLA decreased milk fat synthesis while apparently inhibiting desaturation of 18:0 and 18:2n-6 (Loor and Herbein, 1998). Subsequent infusion studies with trans-10,cis-12 CLA revealed that the reduction in milk delta-9 desaturation ratios coincided with a decrease in SCD1 mRNA abundance (Perfield et al., 2006, 2007). Subsequent molecular research identified negative effects of oleic, linoleic, and linoleic acids on goat mammary gland SCD activity (Bernard et al., 2005, 2009). More recent in vitro research also confirmed that trans-10,cis-12 CLA reduced expression of SCD1 together with SREBF1 (Kadegowda et al., 2009).

From data in ruminant species, it could be concluded that the mechanism whereby PUFA decrease milk fatty acid desaturation ratios involves an inhibition of $S C D 1$ gene transcription or activity. In the present study, PUFA reduced the mRNA level of $S C D 1$ by $50 \%$ and this was paralleled by a $27 \%$ reduction in the expression of SREBF1, which is consistent with previous studies reporting that SREBP mRNA (Xu et al., 1999) and protein (Yahagi et al., 1999) activation are negatively regulated by PUFA. Therefore, taking into account that SREBP could stimulate $S C D 1$ expression (Shimomura et al., 1998; Tabor et al., 1999), PUFA in ruminant mammary cells may modulate $S C D 1$ gene expression through a direct effect on SREBP-1 via several molecular mechanisms.

Polyunsaturated fatty acids are well-known inhibitors of genes containing an SRE binding site in their promoters by decreasing levels of the mature form of the SREBP protein, which presumably occurs from a reduction in the cleavage of the SREBP-1 precursor protein (Worgall et al., 1998; Yahagi et al., 1999). Polyunsaturated fatty acids also could reduce SREBP-1 gene expression by inhibiting insulin-induced protein kinase B phosphorylation (Botolin et al., 2006), which has been proven to activate SREBP-1 expression in rodent liver (Ribaux and Iynedjian, 2003). In bovine Mac-T cells, culture with trans-10, cis-12 CLA decreased lipogenesis through inhibition of the proteolytic activation of SREBP-1 and a subsequence reduction in transcriptional activation of lipogenic genes, including $A C A C A, F A S N$, and SCD1 (Peterson et al., 2004).

Although the effect and regulatory mechanisms induced by PUFA have been well-studied, little is known about the signaling pathways in ruminant mammary cells that might be involved in SCD1 gene transcriptional regulation by PUFA. In our study, it is noteworthy that linoleic acid repressed goat $S C D 1$ promoter activity, which was opposite results reported with bovine Mac-T cells (Keating et al., 2006). One reason

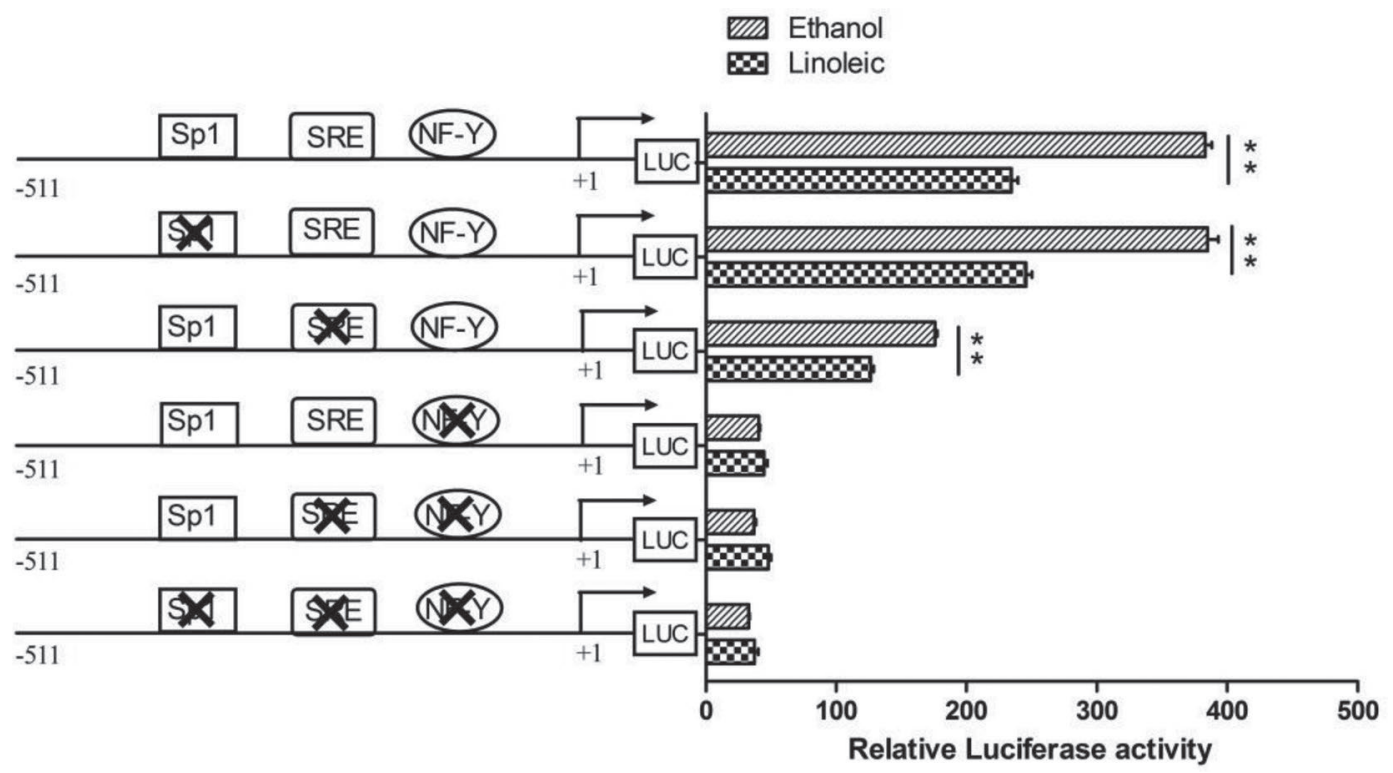

Figure 4. Effects of linoleic acid on site-directed deletion of stearoyl-coenzyme A desaturase 1 (SCD1) promoter activity. Linoleic acid inhibited wild type, Sp1 site-deleted and SRE-deleted SCD1 promoter activity, but had no effect on NF-Y-deleted, both SRE and NF-Y-deleted, and all deleted SCD1 promoter. All experiments were performed in triplicate and repeated 3 times $(\mathrm{n}=9)$. Data are presented as mean \pm SEM for 3 individuals. ${ }^{*} P<0.01$. SRE $=$ sterol response element; $\mathrm{Sp} 1=$ transcription factors specificity protein $1 ; \mathrm{NF}-\mathrm{Y}=$ nuclear factor $\mathrm{Y}$. 
A



B

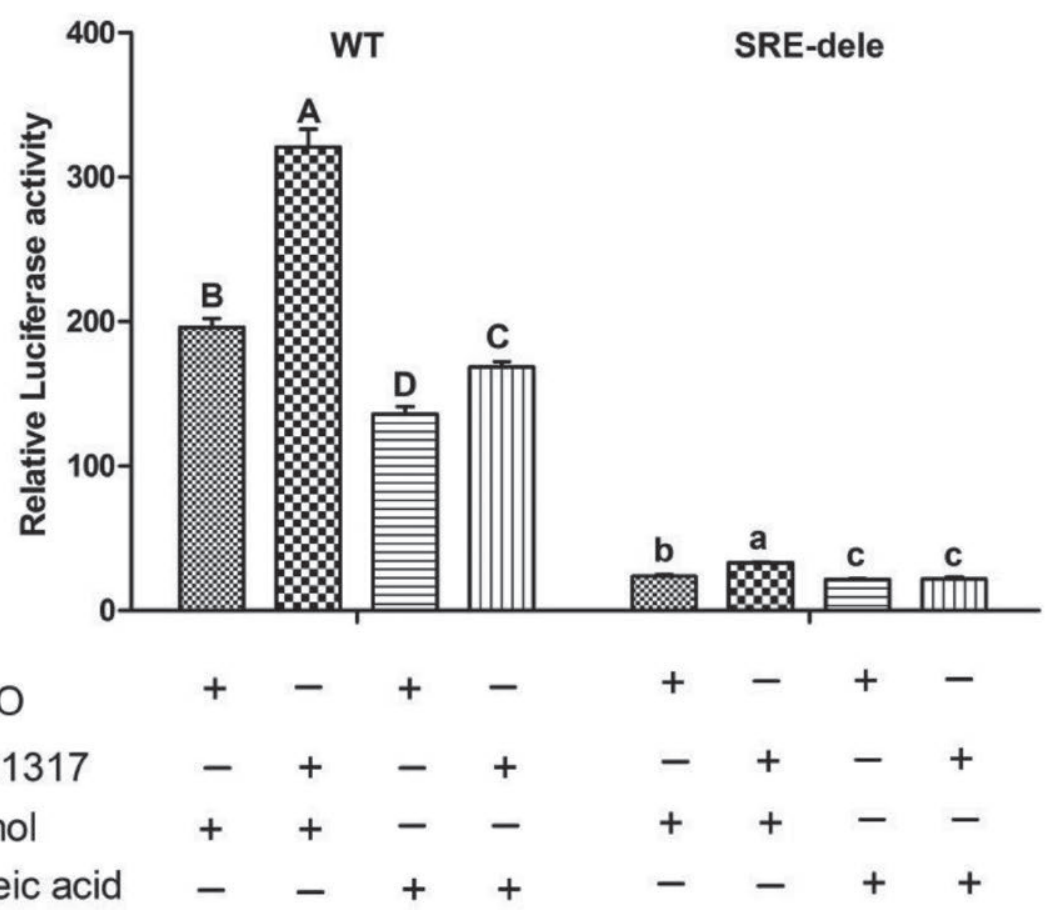

Figure 5. Effects of T0901317 (Sigma-Aldrich, St. Louis, MO) and the combination of linoleic acid treatment on site-directed mutagenesis stearoyl-coenzyme A desaturase 1 (SCD1) promoter activity. (A) Effects of T0901317 on different site-deleted SCD1 promoter activity. Goat mammary epithelial cells (GMEC) were transiently transfected into different site-deleted SCD1 promoters and cultured with T0901317 after $12 \mathrm{~h}$. After $24 \mathrm{~h}$, cells were prepared for luciferase reporter assays. (B) The effect of linoleic acid on wild-type and sterol response element (SRE)-deleted SCD1 promoter activity in response to T0901317. The GMEC were transfected with wild-type or SRE-deleted constructs. After transfection for $12 \mathrm{~h}$, cells were cultured with linoleic acid for $16 \mathrm{~h}$, and then replaced serum-free medium with T0901317. After GMEC were cultured for $24 \mathrm{~h}$, firefly luciferase activity was examined and normalized to Renilla luciferase activity. Values are presented as mean \pm SEM for 3 independent experiments in triplicates $(\mathrm{n}=9)$. ${ }^{*} P<0.05$. Uppercase letters $(A-D)$ indicate significant differences among 4 treatments; lowercase letters $(\mathrm{a}-\mathrm{c})$ denote statistical significance in the SRE-deleted group $(P<0.05)$. DMSO $=$ dimethyl sulfoxide. 
for this contrast could be the fact that Mac-T cells are immortalized whereas the GMEC used in this study were primary cells. In addition, indirect comparison between goat and cows under similar dietary conditions indicated that the SCD enzyme in these species could have different sensitivity (i.e., there appears to be higher sensitivity to linoleic acid inhibition in the goat than cow; Roy et al., 2006; Bernard et al., 2008). The fact that our data agree with a previous study in sheep (Zulkifli et al., 2010) could also indicate that the mammary gland $S C D 1$ in small ruminant species responds in a similar fashion.

In addition, the repressive effect of linoleic acid on $S C D 1$ promoter activity still existed when SRE or Sp1 were mutated alone within the $S C D 1$ promoter region. It is noteworthy that deletion of the nearby NF-Y binding site abolished this effect. In the goat SCD1 gene promoter, a high-affinity CCAAT sequence is juxtaposed to an SRE site, making it possible that SREBP-1 binding to the SRE may be enhanced by NF-Y and the 2 synergize, functionally forming a complex. Such a scenario would allow for bending of the double helix (induced by NF-Y) and might indirectly render the SRE more accessible to DNA binding (Mantovani, 1999). Therefore, SRE deletion may not influence the complex of SREBP-1 with NF-Y, resulting in a PUFA repression of $S C D 1$ promoter activity. It is likely that NF-Y as well as SREBP-1 are also involved in the transcriptional activation of goat $S C D 1$.

Except for a direct effect on SREBP-1, linoleic acid may influence the transcription rate of $S C D 1$ through other mechanisms. For instance, it has been demonstrated that the bovine $S R E B F 1$ promoter contains an SRE that allows for a positive auto-loop regulation by itself, and also contains an LXRE that is responsible for LXR agonist activation (Lengi and Corl, 2010; McFadden and Corl, 2010). Therefore, PUFA could suppress SREBP-1c transcription by inhibition of LXR binding to the LXRE on the SREBF1c promoter $(\mathrm{Ou}$ et al., 2001; Yoshikawa et al., 2002), resulting in downregulated $S C D 1$ gene expression and promoter activity. Although both conserved SRE and LXRE have been characterized in the $S C D 1$ gene promoter in human, mouse, or chicken (Mauvoisin and Mounier, 2011), we did not detect these typical binding sites in the goat $S C D 1$ gene promoter.

It was noteworthy, however, that the $S C D 1$ gene promoter with site-deleted SRE or NF-Y or their combination still could be activated by T09. At the same time, the wild-type $S C D 1$ gene promoter activity also was stimulated by T09 when linoleic acid was included in the culture medium, an effect that is opposite to a study in humans (Caputo et al., 2014). Together with the fact that this effect was abolished once the SRE was deleted, it is possible that novel SRE binding sites may exist because in nonruminants SREBP-1 is a weak activator of transcription and it functions most efficiently only when activated by co-activating transcription factors such as NF-Y and Sp1 (Roder et al., 1999; Bennett and Osborne, 2000; Magaña et al., 2000).

Although our study provided a better understanding of how linoleic acid represses $S C D 1$ gene transcription in goat mammary cells, some limitations exist that need to be considered. For instance, the current model indicates that PUFA likely bind to a putative PUFAbinding protein, leading to repression of transcription upon the binding of the PUFA-binding protein to the PUFA-RR of the SCD1 gene in combination with crosstalk between SREBP-1 and NF-Y (Ntambi, 1999). Therefore, the sophisticated details under which PUFA inhibit $S C D 1$ gene transcription will require further research. Post-transcriptional control of $S C D 1$ by PUFA also might need to be considered. Lastly, the existence of novel LXRE or SRE in SCD1 gene promoter requires further confirmation.

\section{CONCLUSIONS}

A critical region on the $S C D 1$ responsible for linoleic acid repression was identified. Joint or alternate mutations of SRE and NF-Y both decreased the SCD1 gene promoter activity, with NF-Y playing a more essential role. Linoleic acid can decrease $S C D 1$ gene promoter activity and the endogenous expression of $S C D 1$ and $S R E B F 1$. However, the repressive effect induced by linoleic acid is abrogated when NF-Y deleted in $S C D 1$ gene promoter. The LXR ligand T09 activates SCD1 transcription but requires the presence of an intact SRE. Collectively, the data indicate that the mechanism whereby linoleic acid represses $S C D 1$ transcription is mainly through the SRE and NF-Y binding sites in an LXR-dependent fashion.

\section{ACKNOWLEDGMENTS}

This research was jointly supported by the Transgenic New Species Breeding Program of China (Beijing, China; 2014ZX08009-051B) and Special Fund for Agro-scientific Research in the Public Interest (Beijing, China; 201103038).

\section{REFERENCES}

Addrizzo, J. R. 2004. Use of goat milk and goat meat as therapeutic aids in cardiovascular diseases. Dairy Goat Journal 82:27.

Baumgard, L. H., E. Matitashvili, B. Corl, D. Dwyer, and D. Bauman. 2002. Trans-10, cis-12 conjugated linoleic acid decreases lipogenic rates and expression of genes involved in milk lipid synthesis in dairy cows. J. Dairy Sci. 85:2155-2163. 
Bené, H., D. Lasky, and J. M. Ntambi. 2001. Cloning and characterization of the human stearoyl-CoA desaturase gene promoter: transcriptional activation by sterol regulatory element binding protein and repression by polyunsaturated fatty acids and cholesterol. Biochem. Biophys. Res. Commun. 284:1194-1198.

Bennett, M. K., and T. F. Osborne. 2000. Nutrient regulation of gene expression by the sterol regulatory element binding proteins: increased recruitment of gene-specific coregulatory factors and selective hyperacetylation of histone H3 in vivo. Proc. Natl. Acad. Sci. USA 97:6340-6344.

Beppu, F., M. Hosokawa, L. Tanaka, H. Kohno, T. Tanaka, and K. Miyashita. 2006. Potent inhibitory effect of trans-9,trans-11 isomer of conjugated linoleic acid on the growth of human colon cancer cells. J. Nutr. Biochem. 17:830-836.

Bernard, L., C. Leroux, and Y. Chilliard. 2008. Expression and nutritional regulation of lipogenic genes in the ruminant lactating mammary gland. Adv. Exp. Med. Biol. 606:67-108.

Bernard, L., C. Leroux, Y. Faulconnier, D. Durand, K. J. Shingfield, and Y. Chilliard. 2009. Effect of sunflower-seed oil or linseed oil on milk fatty acid secretion and lipogenic gene expression in goats fed hay-based diets. J. Dairy Res. 76:241-248.

Bernard, L., J. Rouel, C. Leroux, A. Ferlay, Y. Faulconnier, P. Legrand, and Y. Chilliard. 2005. Mammary lipid metabolism and milk fatty acid secretion in alpine goats fed vegetable lipids. J. Dairy Sci. 88:1478-1489.

Bhattacharya, A., J. Banu, M. Rahman, J. Causey, and G. Fernandes. 2006. Biological effects of conjugated linoleic acids in health and disease. J. Nutr. Biochem. 17:789-810.

Bionaz, M., and J. J. Loor. 2007. Identification of reference genes for quantitative real-time PCR in the bovine mammary gland during the lactation cycle. Physiol. Genomics 29:312-319.

Botolin, D., Y. Wang, B. Christian, and D. B. Jump. 2006. Docosahexaneoic acid (22:6,n-3) regulates rat hepatocyte SREBP-1 nuclear abundance by Erk- and $26 \mathrm{~S}$ proteasome-dependent pathways. J. Lipid Res. 47:181-192.

Caputo, M., M. C. De Rosa, T. Rescigno, H. Zirpoli, A. Vassallo, N. De Tommasi, G. Torino, and M. F. Tecce. 2014. Binding of polyunsaturated fatty acids to LXRalpha and modulation of SREBP-1 interaction with a specific SCD1 promoter element. Cell Biochem. Funct. 32:637-646.

de Andrade, P. V., and P. Schmidely. 2006. Effect of duodenal infusion of trans-10,cis-12-CLA on milk performance and milk fatty acid profile in dairy goats fed high or low concentrate diet in combination with rolled canola seed. Reprod. Nutr. Dev. 46:31-48.

DeWille, J. W., and S. J. Farmer. 1993. Linoleic acid controls neonatal tissue-specific stearoyl-CoA desaturase mRNA levels. Biochim. Biophys. Acta 1170:291-295.

Espenshade, P. J. 2006. SREBPs: Sterol-regulated transcription factors. J. Cell Sci. 119:973-976.

Gervais, R., J. W. McFadden, A. J. Lengi, B. A. Corl, and P. Y. Chouinard. 2009. Effects of intravenous infusion of trans-10, cis-12 18:2 on mammary lipid metabolism in lactating dairy cows. J. Dairy Sci. 92:5167-5177.

Goldman, P., and P. R. Vagelos. 1961. The specificity of triglyceride synthesis from diglycerides in chicken adipose tissue. J. Biol. Chem. 236:2620-2623.

Haenlein, G. F. W. 2004. Goat milk in human nutrition. Small Rumin. Res. 51:155-163.

Invernizzi, G., B. J. Thering, M. A. McGuire, G. Savoini, and J. J. Loor. 2010. Sustained upregulation of stearoyl-CoA desaturase in bovine mammary tissue with contrasting changes in milk fat synthesis and lipogenic gene networks caused by lipid supplements. Funct. Integr. Genomics 10:561-575.

Kadegowda, A. K., M. Bionaz, L. S. Piperova, R. A. Erdman, and J. J. Loor. 2009. Peroxisome proliferator-activated receptor-gamma activation and long-chain fatty acids alter lipogenic gene networks in bovine mammary epithelial cells to various extents. J. Dairy Sci. 92:4276-4289.

Keating, A. F., J. J. Kennelly, and F. Q. Zhao. 2006. Characterization and regulation of the bovine stearoyl-CoA desaturase gene promoter. Biochem. Biophys. Res. Commun. 344:233-240.
Landschulz, K. T., D. B. Jump, O. A. MacDougald, and M. D. Lane 1994. Transcriptional control of the stearoyl-CoA desaturase-1 gene by polyunsaturated fatty acids. Biochem. Biophys. Res. Commun. 200:763-768.

Lengi, A. J., and B. A. Corl. 2010. Short communication: Identification of the bovine sterol regulatory element binding protein$1 c$ promoter and its activation by liver $\mathrm{X}$ receptor. J. Dairy Sci. 93:5831-5836.

Lin, X. Z., J. Luo, L. P. Zhang, W. Wang, H. B. Shi, and J. J. Zhu. 2013. MiR-27a suppresses triglyceride accumulation and affects gene mRNA expression associated with fat metabolism in dairy goat mammary gland epithelial cells. Gene 521:15-23.

Loor, J. J., and J. H. Herbein. 1998. Exogenous conjugated linoleic acid isomers reduce bovine milk fat concentration and yield by inhibiting de novo fatty acid synthesis. J. Nutr. 128:2411-2419.

Ma, L., and B. A. Corl. 2012. Transcriptional regulation of lipid synthesis in bovine mammary epithelial cells by sterol regulatory element binding protein-1. J. Dairy Sci. 95:3743-3755.

Magaña, M. M., S.-H. Koo, H. C. Towle, and T. F. Osborne. 2000. Different sterol regulatory element-binding protein-1 isoforms utilize distinct co-regulatory factors to activate the promoter for fatty acid synthase. J. Biol. Chem. 275:4726-4733.

Mantovani, R. 1999. The molecular biology of the CCAAT-binding factor NF-Y. Gene 239:15-27.

Mauvoisin, D., and C. Mounier. 2011. Hormonal and nutritional regulation of SCD1 gene expression. Biochimie 93:78-86.

McFadden, J. W., and B. A. Corl. 2010. Activation of liver X receptor (LXR) enhances de novo fatty acid synthesis in bovine mammary epithelial cells. J. Dairy Sci. 93:4651-4658.

Noakes, M., P. J. Nestel, and P. M. Clifton. 1996. Modifying the fatty acid profile of dairy products through feedlot technology lowers plasma cholesterol of humans consuming the products. Am. J. Clin. Nutr. 63:42-46.

Ntambi, J. M. 1999. Regulation of stearoyl-CoA desaturase by polyunsaturated fatty acids and cholesterol. J. Lipid Res. 40:1549-1558.

Ou, J., H. Tu, B. Shan, A. Luk, R. A. DeBose-Boyd, Y. Bashmakov, J. L. Goldstein, and M. S. Brown. 2001. Unsaturated fatty acids inhibit transcription of the sterol regulatory element-binding protein-1c (SREBP-1c) gene by antagonizing ligand-dependent activation of the LXR. Proc. Natl. Acad. Sci. USA 98:6027-6032.

Perfield, J. W., 2nd, P. Delmonte, A. L. Lock, M. P. Yurawecz, and D. E. Bauman. 2006. Trans-10,trans-12 conjugated linoleic acid does not affect milk fat yield but reduces delta9-desaturase index in dairy cows. J. Dairy Sci. 89:2559-2566.

Perfield, J. W., 2nd, A. L. Lock, J. M. Griinari, A. Saebo, P. Delmonte, D. A. Dwyer, and D. E. Bauman. 2007. Trans-9, cis-11 conjugated linoleic acid reduces milk fat synthesis in lactating dairy cows. J. Dairy Sci. 90:2211-2218.

Peterson, D. G., E. A. Matitashvili, and D. E. Bauman. 2004. The inhibitory effect of trans-10,cis-12 CLA on lipid synthesis in bovine mammary epithelial cells involves reduced proteolytic activation of the transcription factor SREBP-1. J. Nutr. 134:2523-2527.

Ramunno, L., G. Cosenza, A. Rando, A. Pauciullo, R. Illario, D. Gallo, D. Di Berardino, and P. Masina. 2005. Comparative analysis of gene sequence of goat CSN1S1 $\mathrm{F}$ and $\mathrm{N}$ alleles and characterization of CSN1S1 transcript variants in mammary gland. Gene 345:289-299.

Ribaux, P. G., and P. B. Iynedjian. 2003. Analysis of the role of protein kinase B (cAKT) in insulin-dependent induction of glucokinase and sterol regulatory element-binding protein 1 (SREBP1) mRNAs in hepatocytes. Biochem. J. 376:697-705.

Roder, K., S. Wolf, K. Larkin, and M. Schweizer. 1999. Interaction between the two ubiquitously expressed transcription factors NF-Y and Sp1. Gene 234:61-69.

Roy, A., A. Ferlay, K. Shingfield, and Y. Chilliard. 2006. Examination of the persistency of milk fatty acid composition responses to plant oils in cows given different basal diets, with particular emphasis on trans-C18: 1 fatty acids and isomers of conjugated linoleic acid. Anim. Sci. 82:479-492.

Shi, H., J. Luo, J. Zhu, J. Li, Y. Sun, X. Lin, L. Zhang, D. Yao, and H. Shi. 2013. PPAR $\gamma$ regulates genes involved in triacylglycerol 
synthesis and secretion in mammary gland epithelial cells of dairy goats. PPAR Res. 2013:310948.

Shimomura, I., H. Shimano, B. S. Korn, Y. Bashmakov, and J. D. Horton. 1998. Nuclear sterol regulatory element-binding proteins activate genes responsible for the entire program of unsaturated fatty acid biosynthesis in transgenic mouse liver. J. Biol. Chem. 273:35299-35306.

Tabor, D. E., J. B. Kim, B. M. Spiegelman, and P. A. Edwards. 1999 Identification of conserved cis-elements and transcription factors required for sterol-regulated transcription of stearoyl-CoA desaturase 1 and 2. J. Biol. Chem. 274:20603-20610.

Teran-Garcia, M., C. Rufo, M. T. Nakamura, T. F. Osborne, and S. D. Clarke. 2002. NF-Y involvement in the polyunsaturated fat inhibition of fatty acid synthase gene transcription. Biochem. Biophys. Res. Commun. 290:1295-1299.

Ulven, S. M., K. T. Dalen, J. A. Gustafsson, and H. I. Nebb. 2005. LXR is crucial in lipid metabolism. Prostaglandins Leukotr. Essent. Fatty Acids 73:59-63.

Wang, W., J. Luo, Y. Zhong, X. Z. Lin, H. B. Shi, J. J. Zhu, J. Li, Y. T. Sun, and W. S. Zhao. 2012. Goat liver X receptor alpha, molecular cloning, functional characterization and regulating fatty acid synthesis in epithelial cells of goat mammary glands. Gene 505:114-120.

Waters, K. M., C. W. Miller, and J. M. Ntambi. 1997. Localization of a polyunsaturated fatty acid response region in stearoyl-CoA desaturase gene 1. Biochim. Biophys. Acta 1349:33-42.

Williams, C. M. 2000. Dietary fatty acids and human health. Ann. Zootech. 49:165-180.

Worgall, T. S., S. L. Sturley, T. Seo, T. F. Osborne, and R. J. Deckelbaum. 1998. Polyunsaturated fatty acids decrease expression of promoters with sterol regulatory elements by decreasing levels of mature sterol regulatory element-binding protein. J. Biol. Chem. 273:25537-25540.

Xu, J., M. T. Nakamura, H. P. Cho, and S. D. Clarke. 1999. Sterol regulatory element binding protein-1 expression is suppressed by dietary polyunsaturated fatty acids. A mechanism for the coordinate suppression of lipogenic genes by polyunsaturated fats. J. Biol. Chem. 274:23577-23583.

Yahagi, N., H. Shimano, A. H. Hasty, M. Amemiya-Kudo, H. Okazaki, Y. Tamura, Y. Iizuka, F. Shionoiri, K. Ohashi, J. Osuga, K. Harada, T. Gotoda, R. Nagai, S. Ishibashi, and N. Yamada. 1999. A crucial role of sterol regulatory element-binding protein-1 in the regulation of lipogenic gene expression by polyunsaturated fatty acids. J. Biol. Chem. 274:35840-35844.

Yoshikawa, T., H. Shimano, N. Yahagi, T. Ide, M. Amemiya-Kudo, T. Matsuzaka, M. Nakakuki, S. Tomita, H. Okazaki, Y. Tamura, Y. Iizuka, K. Ohashi, A. Takahashi, H. Sone, J. Osuga Ji, T. Gotoda, S. Ishibashi, and N. Yamada. 2002. Polyunsaturated fatty acids suppress sterol regulatory element-binding protein 1c promoter activity by inhibition of liver X receptor (LXR) binding to LXR response elements. J. Biol. Chem. 277:1705-1711.

Zhang, L., L. Ge, T. Tran, K. Stenn, and S. Prouty. 2001. Isolation and characterization of the human stearoyl-CoA desaturase gene promoter: requirement of a conserved CCAAT cis-element. Biochem. J. 357:183-193.

Zhu, J., Y. Sun, J. Luo, M. Wu, J. Li, and Y. Cao. 2015. Specificity protein 1 regulates gene expression related to fatty acid metabolism in goat mammary epithelial cells. Int. J. Mol. Sci. 16:18061820

Zulkifli, R. M., T. Parr, A. M. Salter, and J. M. Brameld. 2010. Regulation of ovine and porcine stearoyl coenzyme A desaturase gene promoters by fatty acids and sterols. J. Anim. Sci. 88:2565-2575. 